Pakistan Journal of Neurological Sciences (PJNS)

Volume 8 Issue 4

Article 3

$12-2013$

\title{
Chronic Relapsing Inflammatory Optic Neuropathy (Crion)
}

Muhammad Athar Athar Javed

King Edward Medical University, Lahore

Adnan Hameed Gill Dr

King Edward Medical University, Lahore

Ahmad Furqan Dr

King Edward Medical University, Lahore

Follow this and additional works at: https://ecommons.aku.edu/pjns

Part of the Neurology Commons

\section{Recommended Citation}

Athar Javed, Muhammad Athar; Hameed Gill, Adnan Dr; and Furqan, Ahmad Dr (2013) "Chronic Relapsing Inflammatory Optic Neuropathy (Crion)," Pakistan Journal of Neurological Sciences (PJNS): Vol. 8 : Iss. 4 , Article 3.

Available at: https://ecommons.aku.edu/pjns/vol8/iss4/3 


\section{CHRONIC RELAPSING INFLAMMATORY OPTIC NEUROPATHY $(\mathrm{CRION})$}

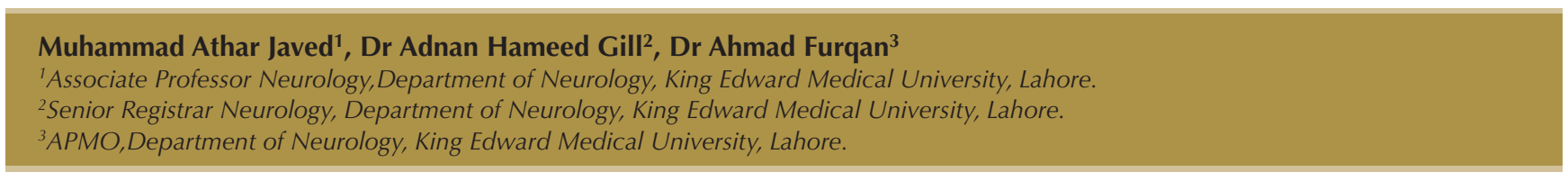

Correspondence to: Mohammad AtharJaved, Associate Professor Neurology, Department of Neurology, King Edward Medical University, Lahore. Email: dratharjaved@hotmail.com.

\section{ABSTRACT}

Background: This newly recognized entity named chronic relapsing inflammatory optic neuropathy (CRION) is a form of inflammatory optic neuropathy which is frequently bilateral and often painful, and is characterized by relapses and remissions. There is no evidence of acquired demyelinating disorders and systemic collagen vascular or granulomatous diseases. Objective: To study the clinical features and response to treatment in patients with recurrent optic neuritis consistent with diagnosis of chronic relapsing inflammatory optic neuropathy (CRION). Materials and Methods: This is retrospective cross sectional study carried out at department of Neurology, King Edward Medical University, Lahore. Patients with history of recurrent episodes of acute or subacute loss of vision accompanied by pain consistent with optic neuritis, unilateral or bilateral, but, without evidence of an acquired demyelinating disorders, systemic collagen vascular orgranulomatous diseases were included in the study. The response to various treatments was also analyzed.Results: A total of 4 patients were identified. All were females with mean age at presentation 35.50+8.10 years and mean age at onset of first episode was $28+10.92$ years. Mean duration of illness was $7.25+4.57$ years. The number of episodes varied from 3-6 (mean 4+1.41episodes). Mean episodes of right optic neuritis were 2.25+0.95 and left side $1.50+0.57$. Only one patient had an episode of simultaneous bilateral optic neuritis. All patients experienced severe pain with loss of vision to finger counting at less than one meter. MRI brains, detailed vasculitis profile, serum angiotensin converting enzyme (ACE) inhibitor level, and X-ray chests were normal in all patients. The CSF for oligoclonal band was also negative. All patients received pulse therapy with methylprednisolone $1 \mathrm{gm}$ daily for three days followed by two weeks oral taper. Most patients improved after first episode but there was partial recovery after the second and subsequent episodes. Only one patient had complete loss of vision in one eye. Two patients received long term oral steroids and azathioprine with prevention of further relapses. One patient received beta interferonwith complete remission so far. One patient received cyclosporine and oral steroids with remission for 6 years but subsequently relapsed after immunotherapy was discontinued. Conclusion: Chronic relapsing inflammatory optic neuropathy (CRION) should be considered in patients with history of recurrent optic neuritis after exclusion of acquired demyelinating disorders, and systemic collagen vascular or granulomatous diseases.

Keywords: Recurrent optic neuritis, Demyelinating disorders, Steroids, Immunosuppressive therapy

\section{INTRODUCTION}

Optic neuritis $(\mathrm{ON})$ is an inflammatory optic neuropathy characterized by loss of vision and may be associated with eye pain. It can affect either or both eyes, sequentially or simultaneously. It is most commonly associated with acquired demyelinating disorders such as multiple sclerosis (MS), neuromyelitisoptica (NMO), systemic connective tissue or granulomatous disease such as sarcoidosis. Other causes such as various toxic, nutritional, metabolic, vascular, hereditary, infectious, and infiltrative etiologies may be associated with optic neuropathy. Diagnosis of recurrent optic neuritis may be difficult and challenging. The accurate treatment and prediction of the prognosis depends on the correct diagnosis.

The newly recognized entity named chronic relapsing inflammatory optic neuropathy (CRION) is a form of inflammatory optic neuropathy which is frequently bilateral and often painful, and is characterized by relapses and remissions. The condition was first time described by Kidd et al in 2003. ${ }^{1}$ Neuroimaging is normal and cerebrospinal fluid examination for oligoclonal band is negative. The syndrome behaves in a way which is typical of the condition known as granulomatous optic neuropathy, but, without systemic evidence of 
sarcoidosis. ${ }^{2-6}$ The diagnosis requires exclusion of all causes as mentioned above. We under took this study to evaluate the clinical features and response to treatment in patients with recurrent optic neuritis consistent with diagnosis of chronic relapsing inflammatory optic neuropathy (CRION).

\section{MATERIALS AND METHODS}

This is retrospective cross sectional study carried out at department of Neurology, King Edward Medical University,Lahore. Study period was 7 years from April 2006 till March 2013. Optic neuritis (ON) consistent with CRION was diagnosed if patient met the following criteria: 1) Recurrent episodes of acute or subacute loss of vision associated with pain with one or more of the following features: a)Relative afferent pupillary defect (unilateral cases); b)Visual field deficit or scotoma; c) Impaired color vision; d) Abnormal visual evoked potentials. 2) Normal MRI brain and / or spinal cord. 3) Normal CSF examination with negativeoligoclonal band and no clinical or laboratory evidence of either systemic vasculitis or sarcoidosis. Recurrent optic neuritis (RON) was defined as a new attack (Unilateral or bilateral) occurring after an interval of 4 weeks. Simultaneous bilateral optic neuritis (SBON) was defined as involvement of both eyes simultaneously or within 4 weeks. Bilateral optic neuritis (BON) if both eyes were involved separately after an interval of 4 weeks. Recurrent unilateral ON (UON) was defined as recurrent episodes of ON involving same eye. Remission was defined as partial or complete recovery from baseline maximal deficit before treatment.Inclusion criteria included following: a) Patients with history of recurrent optic neuritis consistent with CRION as defined above ; b)Age $>13$ years; c)Both sexes; d) Normal neuroimaging of brain and /or spinal cord; and e) CSF -ve for oligoclonal bands. Exclusion criteria were: a) Age < 13years; b) abnormal MRI brain or spinal cord; c) clinical features consistent with systemic vasculitis or sarcoidosis ; d) + ve serology for systemic vasculitis or sarcoidosis. Clinical, laboratory features and treatment received by the patients were recorded on a performa. Data was analyzed using SPSS version 19.

\section{RESULTS}

A total of 4 patients were identified. All were females with mean age at presentation $35.50+8.10$ years and mean age at onset of first episode was $28+10.92$ years. Mean duration of illness was $7.25+4.57$ years(Table 1). The number of episodes varied from 3-6 (mean 4+1.41episodes). Mean episodes of right optic neuritis were $2.25+0.95$ and left side
$1.50+0.570$ nly one patient had an episode of simultaneous bilateral optic neuritis. The all patients experienced severe pain with loss of vision to finger counting at less than one meter. MRI brain, detailed vasculitis profile, serum angiotensin converting enzyme (ACE) inhibitor level, and X-ray chest were normal. The CSF for oligoclonal band was also negative. All patients received pulse therapy with methylprednisolone $1 \mathrm{gm}$ daily for three days followed by two weeks oral taper. Most patients improved after first episode but there was partial recovery after the second and subsequent episodes. Only one patient had complete loss of vision in one eye. Two patients received long term oral steroids and azathioprine with prevention of further relapses. One patient received beta interferon with complete remission so far. One patient received cyclosporine and oral steroids with remission for 6 years but subsequently relapsed after immunotherapy was discontinued.

\section{DISCUSSION}

This is the first study on CRION from Pakistan. The recognition of this entity is important because further episode of optic neuritis can be prevented by use of long term steroids and immunosuppressive therapy. The condition appeared to be more common in females. In our study all patients were female. This female dominance has been reported in other studies as well. ${ }^{7}$ The mean age at the onset of optic neuritis in our study was $28+10.92$ years(range 15 - 38 yrs) compared with 34 years (range 19 - 54 yrs) in the study by Kidd et al. ${ }^{1}$ The mean age at onset was 30 years in another study. ${ }^{8}$

It is difficult to diagnose CRION after first episode of optic neuritis because there is no laboratory hallmark of this condition as yet. How ever certain clinical features may help suspect CRION. Pain is more severe and prolonged in CRION than in demyelinating disorders and persists even after onset of visual loss. ${ }^{1}$ In our study all patients experienced pain that persisted for variable time after the onset of visual loss. Another important feature was excellent response to steroids and recurrence of symptoms after discontinuation of steroids. One of our patients developed relapse when immunosuppressive therapy was discontinued after six years indicating that these patients may require lifelong maintenance therapy. Similar experiences have been observed in other studies. ${ }^{1,9}$ Abrupt steroid withdrawal has been found to be related to irreversible visual loss.${ }^{1}$ In our study extensive work up for acquired demyelinating disorders, and systemic connective tissue or granulomatous disease was negative which is in accordance with all studies reporting CRION so far. ${ }^{9}$ Mean duration of illness was $7.25+4.57 \mathrm{yrs}$ in our study and none of the 
patients developed any clinical or laboratory feature of acquired demyelinating disorders, systemic connective tissue or granulomatous disease.

There had been attempts to discover some laboratory markers for the diagnosis of CRION but have been unsuccessful. ${ }^{10}$

\section{CONCLUSION}

Chronic Relapsing Inflammatory Optic Neuropathy (CRION) is a distinct entity and needs careful recognition as permanent visual loss can be prevented with appropriate management.

Table1. Clinical features of optic neuritis (ON)

\begin{tabular}{|c|c|c|c|c|c|c|}
\hline Cases & Gender & $\begin{array}{l}\text { Age at the onset } \\
\text { of ON (yrs) }\end{array}$ & $\begin{array}{l}\text { Duration of } \\
\text { Illness (yrs) }\end{array}$ & $\begin{array}{l}\text { Number of } \\
\text { episodes } \\
\text { of ON }\end{array}$ & $\begin{array}{l}\text { Time interval between } \\
\text { Attacks yrs }(y), \\
\text { months }(m)\end{array}$ & $\begin{array}{l}\text { Pain associated } \\
\text { with ON }\end{array}$ \\
\hline Case1 & $\mathrm{F}$ & 15 & 14 & 3 & $5 Y, 8 Y$ & + \\
\hline Case 2 & $\mathrm{~F}$ & 36 & 6 & 6 & $6 m, 4 m, 3 m, 4 m, 2 m$ & + \\
\hline Case 3 & $F$ & 38 & 5 & 3 & $1 \mathrm{Y}, 6 \mathrm{~m}, 3 \mathrm{~m}$ & + \\
\hline Case 4 & $F$ & 23 & 4 & 5 & $2 \mathrm{Y}, 1 \mathrm{Y}, 3 \mathrm{~m}, 2 \mathrm{~m}, 2 \mathrm{~m}$ & \\
\hline Total & & mean $=28 \pm 10.92$ & mean $=7.25+4.57$ & 17 & Mean $=16.93+27.46 \mathrm{~m}$ & + in all \\
\hline
\end{tabular}

\section{REFERENCES}

1. Kidd D, Burton B, Plant GT, Graham EM: Chronic Inflammatory Optic Neuropathy. Brain 2003, 126:276-284.

2. Frisen L, Lindgren S, MacGregor BJ, Stattin S. Sarcoid-like disorder of the intracranial optic nerve. J NeurolNeurosurg Psychiatry 1977; 40: 702-7.

3. Galetta S, Schatz NJ, Glaser JS. Acute sarcoid optic neuropathy with spontaneous recovery. J ClinNeuroophthalmol 1989; 9: 27 - 32.

4. Graham EM, Ellis CJ, Sanders MD, McDonald WI. Optic neuropathy in sarcoidosis. J NeurolNeurosurg Psychiatry 1986; 49: 756-63.

5. Gudeman SK, Selhorst JB, Susac JO, Waybright EA. Sarcoid optic neuropathy. Neurology 1982; 32: $597 \pm 603$.

6. Ing EB, Garrity JA, Cross SA, Ebersold MJ. Sarcoid masquerading as optic nerve sheath meningioma. [Review]. Mayo ClinProc 1997; 72: 38-43.

7. Kurne A, Krabudak R, Cakmakli GY et al (2010) Recurrent optic neuritis: clues from a long-term follow-up study of recurrent and bilateral optic neuritis patients. Eye Brain 2:15-20.
8. Saini M, Khurana D: Chronic Inflammatory Optic Neuropathy. Ann Indian AcadNeurol 2010, 13:61 63.

9. Petzold A, Plant GT. Chronic relapsing inflammatory optic neuropathy: a systematic review of 122 cases reported J Neurol2013

10. Storoni M, Verbeek MM, Illes Z, Marignier R, Teunissen CE, Grabowska M, Confavreux C, Plant GT, Petzold A. Serum GFAP levels in optic neuropathies. J NeurolSci 2012; 317(1-2): 117-122. 